\title{
Coherent population trapping of an electron spin in a single negatively charged quantum dot
}

\author{
XIAODONG XU1, BO SUN ${ }^{1}$, PAUL R. BERMAN ${ }^{1}$, DUNCAN G. STEEL ${ }^{1 *}$, ALLAN S. BRACKER ${ }^{2}$, \\ DAN GAMMON² AND L. J. SHAM ${ }^{3}$ \\ ${ }^{1}$ The H. M. Randall Laboratory of Physics, The University of Michigan, Ann Arbor, Michigan 48109, USA \\ ${ }^{2}$ The Naval Research Laboratory, Washington DC 20375, USA \\ ${ }^{3}$ Department of Physics, The University of California-San Diego, La Jolla, California 92093, USA \\ *e-mail: dst@umich.edu
}

Coherent population trapping (CPT) refers to the steady-state trapping of population in a coherent superposition of two ground states that are coupled by coherent optical fields to an intermediate state in a three-level atomic system ${ }^{1}$. Recently, CPT has been observed in an ensemble of donor-bound spins in GaAs (ref. 2) and in single nitrogen-vacancy centres in diamond ${ }^{3}$ by using a fluorescence technique. Here, we report the demonstration of CPT of an electron spin in a single quantum dot. The observation demonstrates both the CPT of an electron spin and the successful generation of Raman coherence between the two spin ground states of the electron ${ }^{4-6}$. This technique can be used to initialize, at about a gigahertz rate, an electron spin state in an arbitrary superposition by varying the ratio of the Rabi frequencies between the driving and probe fields. The results show the potential importance of charged quantum dots for a solid-state approach to the implementation of electromagnetically induced transparency ${ }^{7,8}$, slow light ${ }^{9}$, quantum information storage ${ }^{10}$ and quantum repeaters ${ }^{11,12}$.

A critical condition for realizing coherent population trapping (CPT) is to have a pair of stable ground states with a relatively long coherence time compared with the excited-state decay time. An electron spin trapped inside a single quantum dot is a system that meets this requirement and constitutes an excellent opportunity for the realization of CPT. The demonstration of CPT shows the existence of the dark state which is important for various physical phenomena, for example, CPT is the central physics of electromagnetically induced transparency ${ }^{8}$, and the fast change of refractive index can lead to the effect of slow light ${ }^{9}$, assuming an ensemble of identical charged quantum dots is available.

The electron spin inside a quantum dot has been proposed as a qubit for quantum computing owing to its long coherence time compared with fast optical operations ${ }^{13}$. An important step towards optically driven quantum computation in the quantum dot system is to generate electron spin coherence. The usual method of creating electron spin coherence in quantum dots is to use pulsed lasers ${ }^{4-6}$. Here, the demonstration of CPT by measurement of the absorption spectrum is evidence of the creation of electron spin coherence at a single quantum dot level by continuous-wave lasers.
Another critical element for quantum information science is the initial quantum state preparation ${ }^{14}$. Electron spin state initialization has recently been realized in a single quantum dot by optical spin cooling techniques with a high fidelity ${ }^{15,16}$. However, the limitation is that only two possible initial qubit states can be prepared, either spin up or spin down. CPT is a process that generates an arbitrary coherent superposition of electron spin ground states, the probability amplitudes of which can be controlled by varying the ratio of Rabi frequencies between the driving and probe optical fields. Therefore, we can prepare an arbitrary initial qubit state by using the CPT technique. In this scheme, the initialization rate is limited by the excited-state decay rate, which is of the order of $10^{9} \mathrm{~s}^{-1}$ (ref. 15).

The sample under study contains self-assembled InAs quantum dots embedded in a Schottky diode structure, which gives us the ability to control the charging state of the quantum dot ${ }^{15,17}$ (see the Supplementary Information). In the experiment, we set the voltage such that only one electron is trapped inside the quantum dot. At zero magnetic field, the energy level structure for the lowest-lying states of the negatively charged quantum dot can be modelled as shown in the upper left inset of Fig. 1. The electron spin ground states (trion states) are labelled as $| \pm(1 / 2)\rangle(| \pm(3 / 2)\rangle)$, where $| \pm(1 / 2)\rangle(| \pm(3 / 2)\rangle)$ denotes the electron (hole) spin states along the growth axis ${ }^{15}$. The only dipole-allowed transition is from the spin ground state $|(1 / 2)\rangle(|-(1 / 2)\rangle)$ to the trion state $|(3 / 2)\rangle$ $(|-(3 / 2)\rangle)$ with $\sigma^{+}\left(\sigma^{-}\right)$polarized light excitation. As the spin-flip Raman transitions are dipole forbidden, the trion system at zero magnetic field can be considered as a double two-level structure, not possible for the realization of CPT.

To create a three-level lambda system, we apply a magnetic field in the Voigt geometry ( $\mathbf{X}$ axis), perpendicular to the sample growth direction ( $\mathbf{Z}$ axis). As the electron and hole in-plane $g$ factors are non-zero, which are 0.49 and 0.13 for this particular quantum dot, the applied field mixes the spin ground states as well as the trion states. The energy level diagram and the associated selection rules of the trion system are shown in the upper right inset of Fig. 1. The new electron spin eigenstates $|X \pm\rangle$ can be excited to either of the trion states $|T \pm\rangle$ with linearly polarized light ${ }^{15}$. Hence, the 


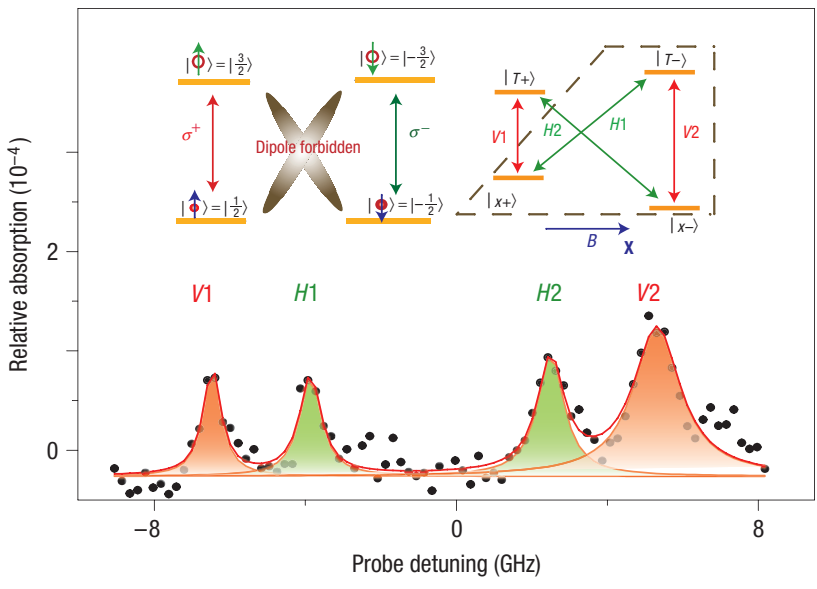

Figure 1 The trion model and its characterization. Inset: Trion energy level diagrams (upper left) without and (upper right) with magnetic field applied in the Voigt geometry. $V(H)$ means the transition is vertically (horizontally) polarized. At zero magnetic field, the spin-flip Raman transitions are dipole forbidden. By applying a magnetic field in the Voigt geometry, the dark transitions become bright. A three-level lambda system is formed by these levels enclosed in the dashed line. The main figure shows the single-beam absorption spectrum of the trion state at a magnetic field of $1.32 \mathrm{~T}$ with $45^{\circ}$ linearly polarized light excitation. A quartet transition pattern is observed as the gate voltage is set in the non-optical pumping region.

forbidden Raman transitions at zero magnetic field are turned on when the magnetic field is applied along the $\mathbf{X}$ axis. As shown in the upper right inset of Fig. 1, we choose $|X \pm\rangle$ and $|T-\rangle$ to form a three-level lambda system. As the transitions from one trion state to the spin ground states are orthogonally polarized, the spontaneously generated coherence that was observed in GaAs interface-fluctuation quantum dots ${ }^{4}$ is absent here.

We first characterized the quantum dot with a single-beam voltage-modulation absorption experiment ${ }^{15,18}$. We set the gate voltage at the edge of the trion charge plateau, where the optical pumping of the electron spin effect is suppressed ${ }^{15,16}$. Figure 1 shows the quartet transition pattern of the trion state using $45^{\circ}$ linearly polarized light excitation at a magnetic field of $1.32 \mathrm{~T}$. The observation of the four transition lines confirms that all four trion transitions are turned on and have similar transition strengths ${ }^{15}$. The four transitions are labelled as $V 1, H 1, H 2$ and $V 2$.

We then set the gate voltage to where the co-tunnellinginduced spin-flip process is suppressed ${ }^{19}$. Figure 2 a shows a singlebeam absorption spectrum by scanning the laser across transition $\mathrm{H} 1$ at a magnetic field of $2.64 \mathrm{~T}$. We observed an almost flat line for the probe absorption spectrum, reflecting the absence of the absorption due to optical pumping ${ }^{15}$. The optical-pumpinginduced saturation of the absorption shows that the spin relaxation rate is much slower than the trion relaxation rate. Hence, the spin ground states can be considered as metastable states compared with the short-lived trion states.

To understand the experimental conditions for the measurements, we consider the interaction scheme shown in Fig. 3. A strong optical field (the driving field) is tuned on resonance with transition $V 2$ and a weak optical field (the probe) is scanned across transition $H 1$. When the probe laser is resonant with transition $H 1$, the two-photon Raman resonance condition is reached. As seen in Fig. $2 \mathrm{~b}$, a clear dip in the probe absorption spectrum is observed for $\Omega_{\mathrm{d}} / 2 \pi=0.56 \mathrm{GHz}$. This observation demonstrates a

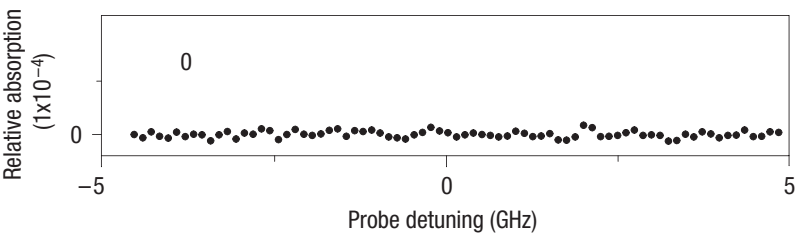

b

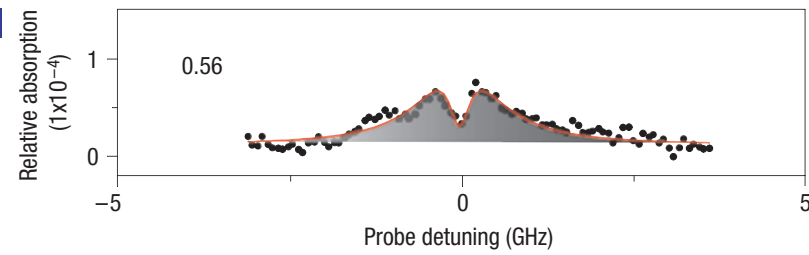

c

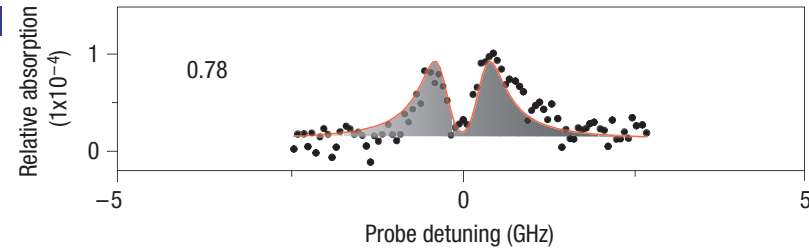

d

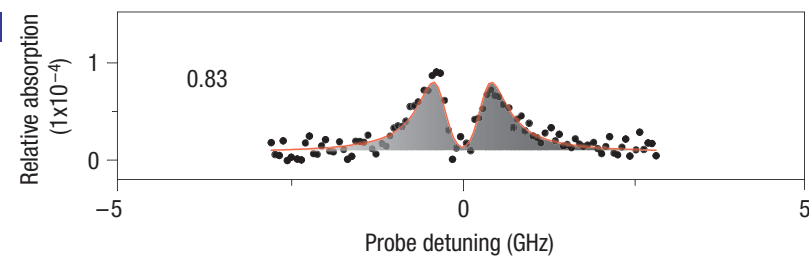

6

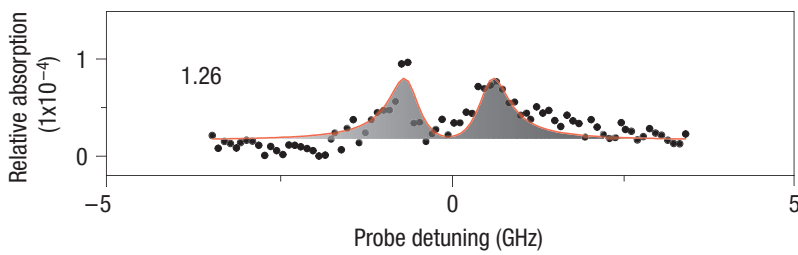

f

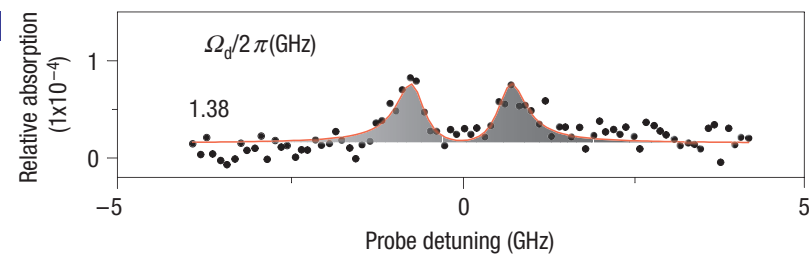

Figure 2 The experimental evidence of the CPT of an electron spin. The gate voltage is set in the optical pumping region and the applied magnetic field is $2.64 \mathrm{~T}$. a, The probe absorption spectrum across transition $H 1$ in the absence of the driving field. $\mathbf{b}-\mathbf{f}$, Probe absorption spectra with various driving-field Rabi frequencies. The driving field is set to be resonant with the transition from $|X-\rangle$ to $|T-\rangle$. The red solid lines are the theoretical fits by solving the optical Bloch equations. $A$ pronounced dip is observed in the probe absorption due to generation of the dark state.

both the generation of the CPT of an electron spin and the Raman coherence between the spin ground states. For this particular set of data, the applied magnetic field is $2.64 \mathrm{~T}$, corresponding to an electron Zeeman splitting of $75.4 \mu \mathrm{eV}(18.2 \mathrm{GHz})$.

The system is described by the optical Bloch equations for the three-level lambda system shown in Fig. 3 , where $\Gamma_{i j}\left(\gamma_{i j}\right)$ is 


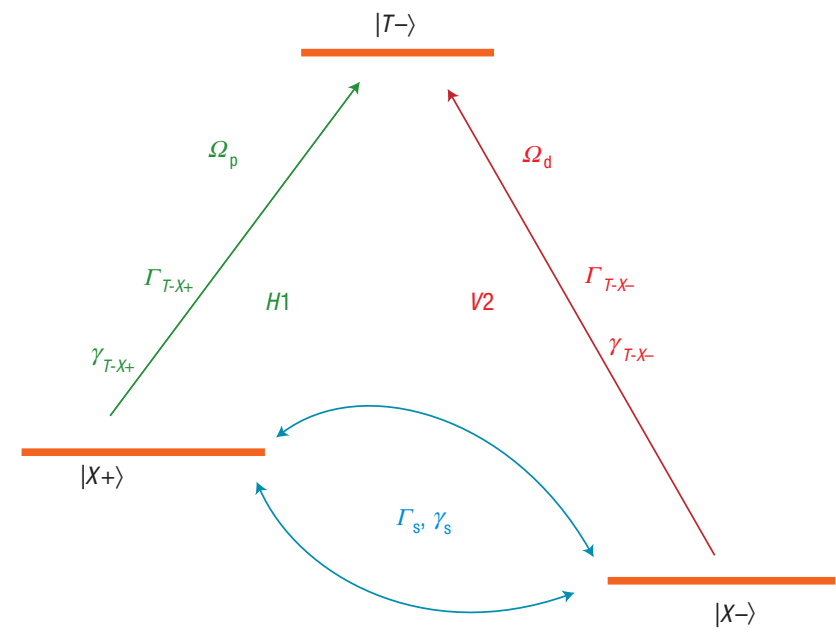

Figure 3 The interaction scheme of the generation of CPT. A three-level lambda system formed by spin ground states $|X \pm\rangle$ of an electron and an intermediate trion state $|T-\rangle$.

the trion population decay (dipole dephasing) rate, $\Gamma_{\mathrm{s}}\left(\gamma_{\mathrm{s}}\right)$ is the electron spin relaxation (decoherence) rate, $\Omega_{i}=\left(\mu_{i} \times E_{i} / \hbar\right)$ is the Rabi frequency, $\mu_{i}$ is the transition dipole moment and $E_{i}$ is the optical field strength. For simplicity, we assume $\Gamma_{\mathrm{s}}, \gamma_{\mathrm{s}} \ll \Gamma_{i j}, \gamma_{i j}, \Omega_{i}$.

The analytical result of the probe absorption spectrum is generally complicated. However, a relatively simple form can be obtained when the driving and probe beams are both on resonance. After simplification, the absorption of the probe beam can be written as

$$
\alpha=\alpha_{o} \frac{\left(\gamma_{\mathrm{s}}-\Gamma_{\mathrm{s}}+\lambda^{2} \Gamma_{\mathrm{s}}\right)}{\left(1+\lambda^{2}\right)^{2}} \frac{\gamma_{T-X+}}{\Omega_{\mathrm{d}}^{2}},
$$

where $\lambda=\left(\Omega_{\mathrm{p}} / \Omega_{\mathrm{d}}\right)$ and $\alpha_{o}$ is a constant. To understand the physics of the dip, we can take $\lambda=1$, the absorption expression is simplified to $\alpha=\left(\alpha_{o} \gamma_{T-X+} / 4\right)\left(\gamma_{\mathrm{s}} / \Omega_{\mathrm{d}}^{2}\right)$. Therefore, the height of the dip is linearly proportional to the spin decoherence rate. If $\gamma_{\mathrm{s}}=0\left(\gamma_{\mathrm{s}} \ll \Omega_{\mathrm{d}}\right)$, the probe absorption vanishes (almost vanishes), that is, the transition becomes transparent to the incident light owing to the destructive interference of the coupled transitions driven by the coherent optical fields.

The observation of CPT can also be understood from the point of view of optical pumping. When the driving and probe lasers are on the two-photon Raman resonance, a coherent dark state is created that is decoupled from the applied optical fields and can be represented as $|D\rangle=\left(\Omega_{d}|X+\rangle-\Omega_{p}|X-\rangle\right) /\left(\sqrt{\Omega_{d}^{2}+\Omega_{p}^{2}}\right)$. Part of the population is excited from the electron spin ground state to the trion state and relaxes spontaneously into the dark state. As the dark state is not 'seen' by the optical fields, the total population is eventually trapped there within a few radiative cycles of the trion state. In CPT, the coherence between the spin ground states is created by the coherent optical fields. Therefore, the whole process is an optical pumping process, the rate of which is ultimately limited by the excited-state decay rate, with the transfer of the mutual coherence between the optical fields to the electron spin coherence.

An arbitrary initial state for the quantum computation can be prepared by varying the ratio of the Rabi frequencies between the driving and probe fields. Ultimately, if we set $\Omega_{\mathrm{d}}$ to zero, the initialized spin state will be $|X-\rangle$. This is the fast spin-state a

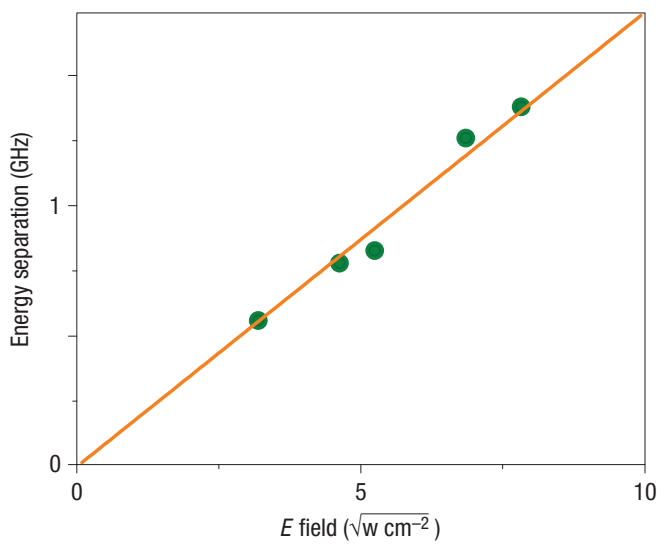

b

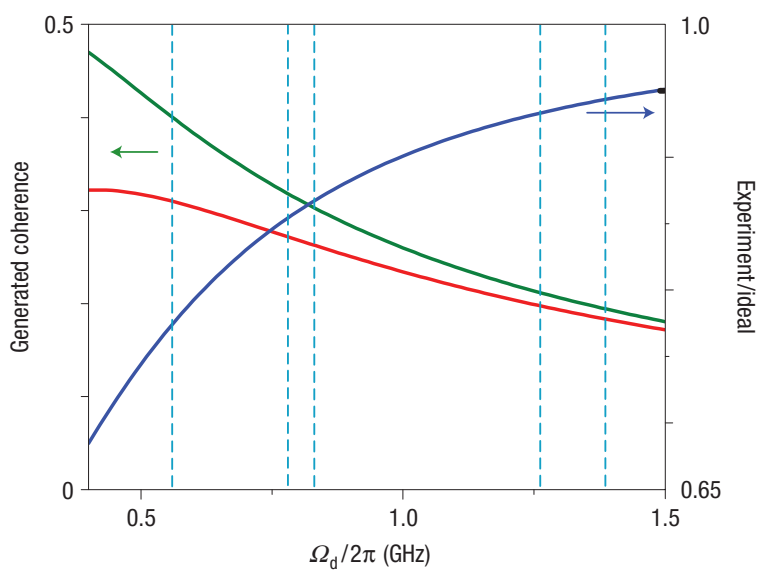

Figure 4 The analysis of the CPT effect. a, The energy separation of the Autler-Townes doublets as a function of driving-field strength. $\mathbf{b}$, Theoretical curves of the creation of the electron spin coherence in a single charged quantum dot. Red line: experimentally generated electron spin Raman coherence $\left(\rho_{X+X_{-}}\right)$inferred from the optical Bloch equations calculation by using the experimental parameters. The calculation is done under the experimental condition that the driving and probe fields are resonant with transition $V 2$ and $H 1$, respectively. Green line: the calculated maximum electron spin Raman coherence in the absence of the electron spin dephasing. Blue line: the ratio of the calculated coherence with and without electron spin dephasing.

preparation effect as discussed in ref. 15. The difference is that when $\Omega_{\mathrm{d}}$ is zero, there is no coherence involved in the state initialization, and the preparation efficiency is determined by the electron spin relaxation rate. In the initialization of the coherent superposition state, we generate an electron spin coherence by the optical fields, and the state preparation efficiency is limited by the electron spin decoherence rate.

The linewidth of the dip in the probe absorption spectrum is ultimately limited by the electron spin decoherence rate. In the experiment, the smallest $\Omega_{\mathrm{d}}$ we applied is $0.56 \mathrm{GHz}$, which is about half of the trion transition linewidth, but still much larger than $\gamma_{s}$. Hence, the linewidth of the dip is broadened by the laser power. When $\Omega_{\mathrm{d}}$ is strong, it will dress the spin ground state $|X-\rangle$ and the trion state $|T-\rangle$. In the case where $\Omega_{\mathrm{d}}$ is larger than the trion transition linewidth, the absorption spectrum of the probe beam will split into two peaks when scanning across transition $H 1$, which are known as Autler-Townes doublets ${ }^{20}$, and has been demonstrated in a neutral quantum $\operatorname{dot}^{21,22}$. The spectral features of the probe absorption spectrum in our experiment are a 
combination of the Autler-Townes splitting and the CPT quantum interference effect ${ }^{7}$, where the spectral positions of the side bands can be determined by the Autler-Townes splitting and the central feature in the absorption spectrum is due to the CPT effect, not a simple summation of the tails of the Autler-Townes Lorentzian line shapes.

The probe absorption spectra with various driving field and fixed probe Rabi frequencies are shown in Fig. 2b-f. The energy separation of the two peaks is increased by increasing the driving field intensity. As $\Omega_{\mathrm{d}}$ becomes larger than the trion transition linewidth, two Autler-Townes peaks with Lorentzian line shapes appear in the probe absorption spectrum, as shown in Fig. 2e,f. Figure $4 \mathrm{a}$ shows the energy separation of the Autler-Townes splitting peaks as a function of the driving field strength. A linear regression fits the data and extends to zero in the absence of the driving field, which indicates that the splitting is dominated by $\Omega_{\mathrm{d}}$. The red solid lines on top of the data shown in Fig. $2 b-f$ are the theoretical fits obtained by solving the optical Bloch equations to all orders in the driving field and to first order in the probe. Assuming that $\gamma_{\mathrm{s}}$ is a few orders of magnitude larger than $\Gamma_{\mathrm{s}}$ (as we show below), we find $\gamma_{T-X+} / 2 \pi$, and $\gamma_{s} / 2 \pi$ equal to $(0.54 \pm 0.1) \mathrm{GHz}$ and $(40 \pm 12) \mathrm{MHz}$, respectively. The value of $40 \mathrm{MHz}$ corresponds to the electron spin decoherence time $T_{2}^{*}\left(1 / \gamma_{\mathrm{s}}\right)$ of $4 \mathrm{~ns}$. Although we measure an electron spin trapped inside a single quantum dot, the electron spin $T_{2}^{*}$ extracted from the data is not the intrinsic electron spin decoherence time owing to the hyperfine interaction between the electron spin and the neighbouring nuclei ensemble ${ }^{23-27}$. The intrinsic $T_{2}$ can be measured by spin $e^{2} o^{28}$ or mode locking of spin coherence techniques ${ }^{5}$.

The generation of the dark state is accompanied by the excitation of the electron spin coherence, which corresponds to the density matrix element $\rho_{X+X-}$. We inserted the parameters extracted from the fits into the optical Bloch equations and obtained values for the coherence between the spin ground states, which are represented by the red line in Fig. $4 \mathrm{~b}$. The green line in Fig. $4 \mathrm{~b}$ represents the theoretical values for the coherence in the absence of spin decoherence, given by $\Omega_{\mathrm{d}} \Omega_{\mathrm{p}} /\left(\Omega_{\mathrm{d}}^{2}+\Omega_{\mathrm{p}}^{2}\right)$. The blue line represents the ratio of the experimentally generated coherence to the ideal case. The light blue dashed vertical lines indicate the applied $\Omega_{\mathrm{d}}$ in the experiment. At the maximally applied Rabi frequency $1.38 \mathrm{GHz}$, we infer that $94 \%$ of the optimal coherence is generated in our system.

Our results open the way to the demonstration of numerous quantum phenomena in spin-based semiconductor quantum dot systems. A direct step is to demonstrate electron spin ground-state Rabi splitting by introducing a third continuous-wave laser, which is an analogy of electron spin Rabi oscillations in the time domain.
Received 29 March 2008; accepted 27 June 2008; published 17 August 2008.

References

1. Gray, H. R., Whitley, R. M. \& Stroud, C. R. Jr. Coherent trapping of atomic populations. Opt. Lett. 3, 218-220 (1978).

2. Fu, K.-M. C., Santori, C., Stanley, C., Holland, M. C. \& Yamamoto, Y. Coherent population trapping of electron spins in a high-purity n-type GaAs semiconductor. Phys. Rev. Lett. 95, 187405 (2005).

3. Santori, C. et al. Coherent population trapping of single spins in diamond under optical excitation. Phys. Rev. Lett. 97, 247401 (2006).

4. Dutt, M.V. G. et al. Stimulated and spontaneous optical generation of electron spin coherence in charged GaAs quantum dots. Phys. Rev. Lett. 94, 227403 (2005).

5. Greilich, A. et al. Mode locking of electron spin coherences in singly charged quantum dots. Science 313, 341-345 (2006).

6. Mikkelsen, M. H., Berezovsky, J., Stoltz, G. N., Coldren, L. A. \& Awschalom, D. D. Optically detected coherent spin dynamics of a single electron in a quantum dot. Nature Phys. 3, 770-773 (2007).

7. Boller, K. J., Imamoglu, A. \& Harris, S. E. Observation of electromagnetically induced transparency. Phys. Rev. Lett. 66, 2593-2596 (1991)

8. Harris, S. E. Electromagnetically induced transparency. Phys. Today 50 (7), 36-42 (1997).

9. Hau, L. V., Harris, S. E., Dutton, Z. \& Behroozi, C. H. Light speed reduction to 17 metres per second in an ultracold atomic gas. Nature 397, 594-598 (1999).

10. Liu, C., Dutton, Z., Behroozi, C. H. \& Hau, L. V. Observation of coherent optical information storage in an atomic medium using halted light pulses. Nature 409, 490-493 (2001).

11. Briegel, H. J., Duer, W., Cirac, J. I. \& Zoller, P. Quantum repeaters: The role of imperfect local operations in quantum communication. Phys. Rev. Lett. 81, 5932-5935 (1998).

12. Duan, L. M., Lukin, M. D., Cirac, J. I. \& Zoller, P. Long-distance quantum communication with atomic ensembles and linear optics. Nature 414, 413-418 (2001).

13. Loss, D. \& DiVincenzo, D. P. Quantum computation with quantum dots. Phys. Rev. A 57, 120-126 (1998).

14. DiVincenzo, D. P. The physical implementation of quantum computation. Fortschr. Phys. 48, $771-783(2000)$

15. Xu, X. et al. Fast spin state initialization in a singly charged InAs-GaAs quantum dot by optical cooling. Phys. Rev. Lett. 99, 097401 (2007).

16. Atature, M. et al. Quantum-dot spin-state preparation with near-unity fidelity. Science 312, 551-553 (2006).

17. Ware, M. E. et al. Polarized fine structure in the photoluminescence excitation spectrum of a negatively charged quantum dot. Phys. Rev. Lett. 95, 177403 (2005).

18. Alen, B. et al. Stark-shift modulation absorption spectroscopy of single quantum dots. Appl. Phys. Lett. 83, 2235-2237 (2003).

19. Smith, J. M. et al. Voltage control of the spin dynamics of an exciton in a semiconductor quantum dot. Phys. Rev. Lett. 94, 197402 (2005).

20. Autler, S. H. \& Townes, C. H. Stark effect in rapidly varying fields. Phys. Rev. 100, 703-722 (1955).

21. Kamada, H., Gotoh, H., Temmyo, J., Takagahara, T. \& Ando, H. Exciton Rabi oscillation in a single quantum dot. Phys. Rev. Lett. 87, 246401 (2001).

22. Xu, X. et al. Coherent optical spectroscopy of a strongly driven quantum dot. Science 317, 929-932 (2007).

23. Khaetskii, A. V., Loss, D. \& Glazman, L. Electron spin decoherence in quantum dots due to interaction with nuclei. Phys. Rev. Lett. 88, 186802 (2002).

24. Coish, W. A. \& Loss, D. Hyperfine interaction in a quantum dot: Non-Markovian electron spin dynamics. Phys. Rev. B 70, 195340 (2004).

25. Johnson, A. C. et al. Triplet-singlet spin relaxation via nuclei in a double quantum dot. Nature $\mathbf{4 3 5}$, 925-928 (2005)

26. Bracker, A. S. et al. Optical pumping of the electronic and nuclear spin of single charge-tunable quantum dots. Phys. Rev. Lett. 94, 047402 (2005).

27. Wang, Y., Liu, R. B. \& Sham, L. J. Restoring coherence lost to a slow interacting mesoscopic spin bath. Phys. Rev. Lett. 98, 077602 (2007).

28. Petta, J. R. et al. Coherent manipulation of coupled electron spins in semiconductor quantum dots. Science 309, 2180-2184 (2005).

Supplementary Information accompanies this paper on www.nature.com/naturephysics.

Acknowledgements

This work is supported by US ARO, DARPA, AFOSR, ONR, NSA/LPS and FOCUS-NSF.

Author information

Reprints and permission information is available online at http://npg.nature.com/reprintsandpermissions. Correspondence and requests for materials should be addressed to D.G.S. 